\title{
Comunicación y divulgación de ciencia en Brasil: un estudio de la usabilidad del portal MCTIC
}

Tatiana Santos Gonçalves | t.goncalvez@gmail.com Instituto Politécnico de Viseu, Portugal

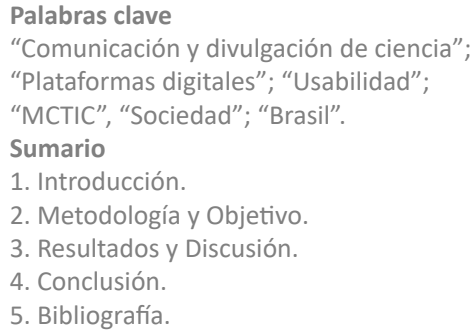

\section{Resumen}

La comunicación y divulgación de la ciencia es un pilar fundamental para entender el proceso de transformación social y económica de un país, ya que la generación de nuevos conocimientos, no pueden traer beneficios a la sociedad, si éstos se mantienen restringidos a un ambiente exclusivamente científico. Esto tiene especial importancia es países en vías de desarrollo como es el

Brasil. En este sentido, el Ministerio de Ciencia, Tecnología, Innovaciones y Comunicaciones, el MCTIC, es el responsable de la implementación y ejecución de programas nacionales para la difusión de la ciencia en Brasil. El portal del MCTIC, es el medio más importante para llevar a cabo tales objetivos. En este trabajo analizaremos la usabilidad de este sitio web, y su efectividad como herramienta para divulgar y promover la adquisición de información sobre ciencia. La usabilidad es uno de los parámetros más importantes para evaluar la efectividad y calidad de un portal, y es esencial para que las plataformas puedan ser utilizadas con éxito. Veremos que, aunque la utilización de esta plataforma digital ofrezca nuevos caminos para la disposición de la información al ciudadano, todavía persiste un retraso significativo en lo que respeta su estructura, especialmente, la usabilidad.

\section{Cómo citar este texto:}

Tatiana Santos Gonçalves (2019): Comunicación y divulgación de ciencia en Brasil: un estudio de la usabilidad del portal MCTIC, en Miguel Hernández Communication Journal, n¹0 (1), pp. 223 a 237. Universidad Miguel Hernández, UMH (Elche-Alicante). DOI: DOI: http://dx.doi.org/10.21134/mhcj.v10i0.293 


\section{Communication and dissemination of science in Brazil: a study of the usability of the MCTIC portal}

Tatiana Santos Gonçalves | t.goncalvez@gmail.com

Instituto Politécnico de Viseu, Portugal

Keywords

"Dissemination of science", "Websites", "Usability",

"MCTIC", "Society", "Brazil".

Summary

1. Introduction.

2. Methodology and Objective.

3. Results and Discussion.

4. Conclusion.

5. Bibliography.

\section{Abstract}

The communication and dissemination of innovation and science is a fundamental pillar to understand the process of social and economic transformation of a country, since the generation of new knowledge, cannot bring benefits to society, if these remain restricted to an

exclusively scientific environment. This is especially important in developing countries such as Brazil. In this sense, the Ministry of Science, Technology, Innovations and Communications, MCTIC, is responsible for the implementation and execution of national programs for the dissemination of science in Brazil. The MCTIC website is the most important way to accomplish these goals. In this work we will analyze this portal and its effectiveness as a tool to disseminate and promote the acquisition of information about science. In particular, this work focuses on the analysis of the usability of the MCTIC portal. Usability is one of the most important parameters to evaluate the effectiveness and quality of the interface of a portal, and it is essential for platforms to be successfully used and citizens can access the contents and services. We will see that, although the use of this digital government platform offers new ways for the provision of information to the citizen, there is still a significant delay in respecting its structure, especially usability.

\section{How to cite this text:}

Tatiana Santos Gonçalves (2019): Communication and dissemination of science in Brazil: a study of the usability of the MCTIC portal, in Miguel Hernández Communication Journal, n¹0 (1), pp. 223 a 237. Universidad Miguel Hernández, UMH (Elche-Alicante). DOI: DOI: http://dx.doi.org/10.21134/mhcj. $\underline{\mathrm{v} 10 \mathrm{i} 0.293}$ 


\section{Introducción}

En el marco de acciones públicas en Brasil para fomentar el diálogo entre científicos y público, se establecieron diversos programas y acciones con miras a la institucionalización de una política nacional para la popularización de la ciencia y de la tecnología en el país (Moreira, 2006). En este contexto, el Ministério da Ciência, Tecnologia, Inovações e Comunicações (Ministerio de Ciencia, Tecnología, Innovaciones y Comunicaciones), el MCTIC, ocupa un lugar central, al ser el máximo responsable de la implementación y ejecución de programas nacionales para la difusión de la ciencia en el país (Brasil, 2016; 2011), siendo su portal web el principal medio para comunicarse con los ciudadanos.

Gracias a la implementación de éste y otros portales de instituciones de la administración del Estado, la sociedad pasó a tener acceso a informaciones públicas con datos y estudios estadísticos fiables (Ferrer y Santos, 2004). Además, los entornos digitales colaborativos facilitan que los ciudadanos puedan interactuar con gestores públicos, por medio de la discusión pública de proyectos, formación de sondeos electrónicos, encuestas online, etc. (Gomes, 2018; Di Gennaro y Dutton, 2006; Dahlgren, 2005; Dahlberg, 2001).

El MCTIC es una de las instituciones gubernamentales brasileñas que han invertido más en la comunicación con el ciudadano a través de plataformas digitales. En su sitio web se ofrece informaciones enfocándose en las acciones en las áreas de la ciencia, tecnología, radio y telecomunicaciones en el país. En sus páginas son presentados contenidos con noticias y publicaciones e informaciones sobre eventos científicos, programas y convocatorias del Ministerio. Además de la disponibilidad a las informaciones públicas y de la oferta de servicios al ciudadano, es importante también entender y analizar la efectividad y la calidad del acceso a las informaciones. Uno de los parámetros más relevantes para estudiar estos asuntos es la usabilidad.

Este estudio tiene como objetivo hacer un análisis de la situación de la divulgación de la ciencia en Brasil. Para alcanzar ese objetivo, este artículo se centró en una evaluación del acceso a los contenidos y servicios del portal MCTIC. En particular, se realizó un análisis de la usabilidad de la interface de ese portal, basado en uno de los métodos más utilizados y aceptados por la comunidad académica: el Método de Evaluación Heurística presentado por Jacob Nielsen (2016b).

\subsection{La comunicación y difusión de ciencia en Brasil y el portal del MCTIC}

A través de la revolución tecnológica y, principalmente, con el advenimiento de las nuevas tecnologías de la información y comunicación (TIC), se han producido un mayor volumen y acceso a la información. De hecho, las TIC proporcionaron un nuevo paradigma en la organización de la información que cambió el método de disponibilidad del contenido informativo (Bohman, 2004).

En el ámbito de la ciencia, los medios y herramientas online ofrecieron una nueva perspectiva para la divulgación del conocimiento científico. El surgimiento de las redes electrónicas de periódicos científicos y de portales con el objetivo de comunicar y divulgar ciencia, reconfiguraron la relación entre ciencia y público. Al mismo tiempo, la expansión de políticas y programas establecidos en diversos contextos, con el objetivo de popularizar la ciencia, refleja el compromiso del Estado con la promoción de la divulgación científica para la sociedad. 
En América Latina, debido a la influencia de los movimientos de popularización de la ciencia en Europa como Public Understand of Science (PUS) y Public Engagement of Science and Technology (PEST), diversas acciones para el estímulo y la participación de la sociedad en las controversias científicas ocurrieron entre los años 1980 y 2000 (Winter, 2004; Bodmer, 1985). De esta manera, políticas y programas para la comunicación y divulgación de la ciencia e innovaciones tecnológicas, involucraron esferas de sector público y privado, como el gobierno, las universidades, los museos, centros de investigación, y medios de comunicación, entre otros (Gonçalves, 2018).

Sin embargo, y a pesar de los avances logrados, la crisis presupuestaria que se impuso a la ciencia en los últimos años en Brasil, (y en algunos otros países de Latinoamérica, como en Venezuela, por ejemplo), abrieron un abismo de comunicación entre la comunidad científica y la sociedad. Y, a pesar de la importancia de la divulgación científica, la discusión sobre la intervención gubernamental para la promoción de la divulgación científica ocupa, actualmente, un lugar modesto en la agenda de los investigadores y de las políticas gubernamentales (Escobar, 2018; Vogt, Gomes \& Muniz, 2018).

Además, la heterogeneidad entre las regiones de Brasil surge como un impedimento más para una mejor divulgación de la ciencia en ese país. La difusión de la ciencia en Brasil fue estudiada por Almeida et al. (2015), Navas y Marandino (2009), Moreira (2006) y Massarani (2002) y se observó que, históricamente existe una tendencia de perpetuación en la heterogeneidad estructural entre las regiones de Brasil, que sólo puede modificarse con políticas activas que reviertan ese retraso. Las regiones norte, nordeste y las zonas rurales son consideradas las zonas más pobres del país, en donde hay una mayor falta de acceso a los bienes materiales y culturales, reflejándose también en un menor conocimiento científico y tecnológico.

De forma que, cerca del $40 \%$ de los brasileños más pobres, no visita museos o centros de ciencia debido a la dificultad del acceso o por no haber en sus ciudades. Según datos de la Associação Brasileira de Centros e Museus de Ciência (Asociación Brasileña de Centros y Museos de Ciencia), en Brasil, hasta el año de 2015, se contabilizaron 268 espacios de divulgación científica, entre museos, centros de ciencias, planetarios, jardines botánicos y zoológicos. De estos espacios, más de la mitad (74\%) se concentra en las regiones sur y sudeste, consideradas las regiones más ricas y desarrolladas del país (Almeida et al., 2015).

Con miras a la institucionalización de una política nacional para la popularización de la ciencia y de la tecnología en el país, se establecieron diversos programas y acciones nacionales (Gonçalves, 2018; Moreira, 2006). En ese escenario, el Ministério da Ciência, Tecnologia, Inovações e Comunicações (Ministerio de Ciencia, Tecnología, Innovaciones y Comunicaciones), el MCTIC, ocupa el papel central. El MCTIC es la institución responsable de los programas para la difusión de la ciencia y también de la radio y la televisión en Brasil.

Este Ministerio ha invertido en proyectos de difusión de conocimiento y tecnologías para comunidades carentes en los medios rural y urbano, con foco en la popularización del conocimiento para la inclusión social (Brasil, 2016; 2011). Con este objetivo, a lo largo de los años se establecieron políticas y estrategias para conseguir una mayor integración y apoyo entre Estados del norte y nordeste y las agencias de fomento, universidades y centros de investigación del sur del país.

Actualmente, ante una nueva realidad social moldeada por las herramientas tecnológicas, el MCTIC 
invirtió en el uso de las herramientas digitales para la accesibilidad de las informaciones y para la comunicación y divulgación de la ciencia en Brasil. En ese contexto, su página web ${ }^{1}$ se convirtió en uno de los principales medios para comunicarse con los ciudadanos.

El portal presenta contenidos con noticias, publicaciones, documentos y servicios, siendo posible encontrar eventos científicos, programas y convocatorias. Se permite al usuario el acceso a galería de imágenes y audios con contenidos sobre actividades del Ministerio, centros de ciencia, universidades y fundaciones públicas en las áreas de la ciencia y tecnología y enlaces para sus redes sociales.

Además, la plataforma dispone de enlaces para bases de datos con documentos, presupuestos, leyes, decretos y medidas gubernamentales del MCTIC en las áreas de la ciencia y la tecnología en el país. Así, los usuarios pueden acceder a datos sobre convenios, gastos, licitaciones, contratos y auditorías realizadas por esta institución. A partir de esta plataforma, también se permite al usuario el acceso a portales de otras instituciones públicas y organismos federales, con consultas públicas sobre las acciones y proyectos realizados por el Gobierno.

A través de la implementación de éste y otros portales de instituciones de la administración del Estado, la sociedad pasó a tener acceso a informaciones públicas con datos y estudios estadísticos fiables (Ferrer y Santos, 2004). Asimismo, los entornos digitales colaborativos facilitan, de manera muy económica, que los ciudadanos puedan interactuar con gestores públicos, por medio de la discusión pública de proyectos, realización de sondeos electrónicos, encuestas online, etc. (Gomes, 2018; Di Gennaro y Dutton, 2006; Dahlgren, 2005; Dahlberg, 2001).

Además de la disponibilidad y la oferta de informaciones, es también importante la efectividad con que se accede a esas informaciones. En este sentido, la usabilidad es unos de los parámetros más estudiados y que puede ayudar a entender y mejorar la comunicación y el acceso a la información pública (Nielsen, 2016b; 2012; Krug, 2013; Hassan y Martín, 2004) por el ciudadano no científico. En este trabajo nos centraremos en el estudio de uno de los aspectos más relevantes de este tipo de plataforma: su usabilidad. En la sección siguiente se analiza la usabilidad de sitios webs con un enfoque en la satisfacción de las necesidades de los usuarios.

\subsection{La usabilidad en los sitios webs}

En términos generales, la usabilidad de un sistema web está estrictamente relacionada con la capacidad de los usuarios para alcanzar metas y objetivos con eficiencia y satisfacción durante la navegación en un sitio web (Nielsen, 2016a; ISO 9142-250, 2010; ISO 9142-11, 2008). Varios estudios, ver por ejemplo Nielsen (2016b; 2012); Krug (2013), Hassan y Martín (2004), han demostrado que el uso de los métodos de usabilidad ayuda significativamente a aumentar la calidad de un sitio web. De hecho, según Nielsen (2012) se estima que un gasto del 10\% del presupuesto en usabilidad duplicaría (en media) las métricas de calidad del sitio web.

Sin embargo, todavía no hay un consenso sobre cómo tratar y evaluar apropiadamente la usabilidad de los sitios web (Lee y Kozar, 2012). Los estudios hechos hasta ahora no son uniformes y están basados en distintos métodos para medir la usabilidad. Cada uno de estos métodos presentan criterios y parámetros diferentes, basados en un conjunto de identificadores que permite evaluar la usabilidad durante los ciclos de implementación y uso.

Investigadores del área de Interacción Hombre-Máquina (IHM) propusieron múltiples factores de usabilidad con el objetivo de medir la calidad de diseño del sitio web. Por ejemplo, Krug (2013) y Delice y Gungor (2009) establecieron como factores para medir la usabilidad: el tiempo en que se carga la página, la eficiencia de la navegación, el tiempo de descarga, la tasa de éxito de búsqueda,

${ }^{1} \mathrm{Al}$ portal del MCTIC se puede acceder en: http://www.mctic.gov.br/portal 
la tasa de errores, el tiempo para completar tareas y la frecuencia del movimiento del cursor. Otros factores de usabilidad propuestos especificaban la facilidad de uso, la facilidad de lectura, la calidad del contenido, el entretenimiento, la productividad y la relevancia (Lee y Kozar, 2012).

Este artículo se centra en uno de los métodos más utilizados y aceptados por la comunidad académica: el Método de Evaluación Heurística presentado por Nielsen (2016b)². A continuación, se presenta en más detalle este método que más tarde será adaptado para la evaluación del acceso a los contenidos y servicios del portal del MCTIC.

\subsection{Evaluación Heurística de Nielsen}

De acuerdo con la definición de Nielsen (2012, s.n), "la usabilidad es un atributo de calidad que evalúa qué tan fáciles de usar son las interfaces de usuario". La palabra "usabilidad" también se refiere a métodos para mejorar la facilidad de uso durante el proceso de diseño.

Para Nielsen (2012) la usabilidad y la utilidad son igualmente importantes y juntos determinan si algo es útil: poco importa que algo sea fácil si no es lo que quieres. Tampoco es bueno si el sistema hipotéticamente puede hacer lo que quiere, pero no puede hacerlo porque la interface es demasiado difícil. Según Nielsen (2012), la usabilidad está definida por cinco componentes de calidad. En su página $w^{3} b^{3}$ se describen estas componentes de la siguiente manera:

- Capacidad de aprendizaje: La facilidad para los usuarios entiendan desde la Página de inicio los contenidos y servicios disponibles a través de la aplicación, y cómo buscar información específica usando los enlaces disponibles para la navegación de hipertexto.

- Eficiencia: Que los usuarios que desean encontrar algunos contenidos pueden comunicarse con ellos rápidamente a través de los enlaces disponibles. Además, cuando los usuarios acceden a una página, deben poder orientarse y comprender el significado de la página con respecto a su punto de inicio de navegación.

- Memorización: implica que, después de un período de no utilización, los usuarios todavía pueden orientarse dentro del hipertexto, por ejemplo, mediante barras de navegación que apuntan a páginas de referencia.

- Errores: Que, en caso de que los usuarios hayan seguido erróneamente un enlace, deberían poder regresar a su ubicación anterior.

- Satisfacción: se refiere a la situación en la cual los usuarios sienten que tienen el control con respecto al hipertexto, gracias a la comprensión de los contenidos disponibles y los comandos de navegación.

Para identificar los problemas de usabilidad, Nielsen (2016b) propuso el Método de Evaluación Heurística. Este método está basado en la verificación de una pequeña lista de reglas que tiene como objetivo descubrir potenciales problemas en la usabilidad del cibermedio. Este método consiste en la evaluación de 10 heurísticas (ítems), con el fin de evitar errores comunes en sitios webs. Nielsen (2016b) describe los parámetros de evaluación heurística de la siguiente manera:

- Visibilidad del status del sistema: El sistema siempre debe mantener informados a los usuarios sobre lo que está sucediendo, a través de comentarios apropiados dentro de un tiempo razonable.

- Compatibilidad entre el sistema y el mundo real: El sistema debe hablar el idioma de los usuarios, con palabras, frases y conceptos familiares para el usuario, en lugar de términos orientados al sistema. Siguiendo las convenciones del mundo real, haciendo que la información aparezca en un orden natural y lógico.

- Control y libertad para el usuario: Los usuarios a menudo eligen las funciones del sistema por error y necesitarán una "salida de emergencia" claramente marcada para abandonar el estado no deseado sin tener que pasar por un diálogo prolongado. Soporte deshacer y rehacer.

${ }^{2}$ La primera versión de este método se presentó en el artículo Enhancing the explanatory power of usability heuristics, en 1994, durante la CH'I94 Conference, en Boston, Estados Unidos. ${ }^{3}$ https://www.nngroup.com/articles/usability-101-introduction-to-usability/ 
- Consistencia y estándares: Los usuarios no deberían preguntarse si las diferentes palabras, situaciones o acciones significan lo mismo.

- Prevención de errores: Un diseño cuidadoso que evita que ocurra un problema en primer lugar es preferible a tener buenos mensajes de error. Elimine las condiciones propensas a errores o verifíquelas y presente a los usuarios una opción de confirmación antes de comprometerse con la acción.

- Reconocimiento en lugar de recuerdo: Minimice la carga de memoria del usuario haciendo visibles objetos, acciones y opciones. El usuario no debería tener que recordar información de una parte del diálogo a otra. Las instrucciones para el uso del sistema deben ser visibles o recuperarse fácilmente cuando corresponda.

- Flexibilidad y eficiencia de uso: Es muy deseable un sistema que pueda atender las necesidades tanto de los usuarios más expertos, como la de los novatos. Permitir a los usuarios personalizar las acciones frecuentes.

- Diseño minimalista y estético: Los diálogos no deben contener información que sea irrelevante o raramente necesaria. Cada unidad adicional de información en un diálogo compite con las unidades de información relevantes y disminuye su visibilidad relativa.

- Ayudar a los usuarios a reconocer, diagnosticar y recuperar errores: Los mensajes de error deben expresarse en lenguaje sencillo (sin códigos), indicando con precisión el problema y sugiriendo, de manera constructiva, una solución.

- Ayuda y documentación: Aunque siempre es mejor que el sistema se pueda usar sin documentación, puede ser necesario proporcionar ayuda y documentación. Cualquier información de este tipo debe ser fácil de buscar, enfocándose en la tarea del usuario, enumerando los pasos concretos que se deben llevar a cabo y no siendo demasiado grande.

Para el estudio de la usabilidad los dos métodos más usados son: las pruebas con los usuarios y el análisis heurístico de expertos. Tal como indica su nombre, la metodología basada en pruebas con los usuarios permite detectar los posibles problemas de usabilidad desde el punto de vista de los sujetos a los que va dirigida la web (Lee y Kozar, 2012). Por otro lado, la segunda se centra en el estudio heurístico a través de interacción con los expertos en usabilidad, donde se evalúan y detectan los posibles problemas de usabilidad. Esta técnica orienta a los evaluadores para inspeccionar de forma sistemática la interfaz de los sistemas y así identificar problemas que comprometen una buena usabilidad.

Para la realización de una evaluación heurística con expertos, Nielsen (2016b) recomiendan de tres a cinco evaluadores como el número de participantes ideal necesario para encontrar problemas de usabilidad. Estos evaluadores pueden identificar alrededor del 75\% al 95\% de los problemas, ya que cada evaluador puede encontrar alrededor del $35 \%$ de los problemas.

\section{Metodología y Objetivo}

Teniendo como objetivo hacer un análisis de la situación de la divulgación de la ciencia en Brasil, a través de la evaluación del acceso a los contenidos y servicios ofrecidos en el portal MCTIC, este estudio se centró en la evaluación de la usabilidad de esta plataforma.

Para llevar a cabo la evaluación, el tipo de metodología utilizada en ese trabajo se basó en un análisis exploratorio y cualitativo (Quivy y Campenhoudt, 2005). En particular, se realizó un análisis de la usabilidad de la interface del portal según el Método de Evaluación Heurística propuesto por Nielsen (2016) . La elección de ese método se debió, principalmente, a su eficiencia en identificar grandes problemas de usabilidad. Así, fueron considerados los parámetros de evaluación heurística, tal y como están descritos en la Sección 1.3. Sin embargo, estos parámetros tuvieron que ser adaptados para el contexto de este trabajo y serán presentados en una planilla de análisis, en la Sección 2.1.

${ }^{4}$ Siguiendo este enfoque propuesto por Nilsen (2016b), no será, a priori, asumida ninguna hipótesis. 
Para este trabajo se optó por una evaluación basada en el análisis de expertos en lugar de una basada en pruebas con los usuarios. Los expertos, todos ellos brasileños, fueron reclutados teniendo en cuenta su nivel de experiencia en evaluación de usabilidad de sitios webs y su conocimiento de los contenidos y servicios disponibles en el cibermedio analizado.

Con base en estos criterios, y siguiendo la recomendación de Nilsen (2016b) de que se utilicen entre tres y cinco evaluadores, fue creado un grupo de tres expertos en usabilidad, constituidos por los siguientes componentes: un diseñador de páginas web, (experto 1), un analista de desempeño interacción humana-computadora (experto 2) y un analista de sistemas (experto 3). Los dos primeros expertos son doctores por la universidad de São Paulo (USP), en el área de ciencias de la información, y el último es doctor por la Universidad de Campinas (UNICAMP), São Paulo, en el área de computación.

\subsection{Procedimiento}

A través de una planilla de análisis (Tabla 1), los expertos evaluaron individualmente la interface del portal MCTIC con el objetivo de identificar los problemas de usabilidad. El procedimiento de evaluación consistió en que cada evaluador inspeccionaba, como mínimo, dos veces la interface del portal: la primera vez para familiarizarse con el contenido, y la segunda vez para hacer la inspección de la página con base en los parámetros de usabilidad escogidos para este estudio.

Tabla 1. Definición de los campos para el análisis formal del fanzine Rockocó. Fuente: Tabla de elaboración propia del autor a partir del estudio planeado.

\begin{tabular}{|l|l|l|}
\hline & \multicolumn{1}{|c|}{ Heurísticas } & $\begin{array}{l}\text { Criterios para la usabilidad de la interface de portal } \\
\text { MCTIC }\end{array}$ \\
\hline 1 & $\begin{array}{l}\text { Visibilidad del status del } \\
\text { sistema }\end{array}$ & $\begin{array}{l}\text { Información de los contenidos presentados en } \\
\text { disponible de forma clara; retroalimentación visual } \\
\text { en la portada; retroalimentación en cada acción del } \\
\text { portal; tiempo apropiado de respuestas a cada tarea; } \\
\text { terminología apropiada de los menús. }\end{array}$ \\
\hline 2 & $\begin{array}{l}\text { Compatibilidad entre el siste- } \\
\text { ma y el mundo real }\end{array}$ & $\begin{array}{l}\text { Iconos objetivos y familiares al usuario; opciones de } \\
\text { menú ordenadas en forma lógica, lenguaje adecuada } \\
\text { al usuario; los colores en la portada corresponden al } \\
\text { sentido común. }\end{array}$ \\
\hline 3 & $\begin{array}{l}\text { Control y libertad para el } \\
\text { usuario }\end{array}$ & $\begin{array}{l}\text { El usuario puede avanzar y retroceder en cada acción } \\
\text { en el portal; los usuarios pueden revertir cada acción } \\
\text { fácilmente; se utilizan menús con muchos niveles en } \\
\text { ese sitio web. }\end{array}$ \\
\hline 4 & Consistencia y estándares & $\begin{array}{l}\text { El sistema sigue las convenciones y expectativas del } \\
\text { usuario; los patrones se utilizaron de la misma mane- } \\
\text { ra en toda la interface; los comandos de la portada se } \\
\text { utilizan de la misma manera. }\end{array}$ \\
\hline
\end{tabular}




\begin{tabular}{|l|l|l|}
\hline 5 & Prevención de errores & $\begin{array}{l}\text { Los errores potenciales se reconocen en el portal; } \\
\text { siempre que sea posible, el sistema en esta platafor- } \\
\text { ma previene al usuario sobre errores; el sistema avisa } \\
\text { al usuario cuando está a punto de cometer un error; } \\
\text { la navegación entre las ventanas del cibermedio } \\
\text { siempre es visible. }\end{array}$ \\
\hline 6 & $\begin{array}{l}\text { Reconocimiento en lugar de } \\
\text { recuerdo }\end{array}$ & $\begin{array}{l}\text { Se utiliza audio para avisar sobre errores en el portal; } \\
\text { los mensajes se muestran en el lenguaje correcto; los } \\
\text { mensajes sugieren la causa del error; la forma de ce- } \\
\text { rrar los mensajes en ese portal es clara; es fácil con- } \\
\text { tactar a los responsables técnicos en caso de error. }\end{array}$ \\
\hline 7 & $\begin{array}{l}\text { Flexibilidad y eficiencia de } \\
\text { uso }\end{array}$ & $\begin{array}{l}\text { El uso de color es consistente en la aplicación; los } \\
\text { menús inactivos quedan visiblemente desactivados } \\
\text { o se omiten; los títulos y enlaces se describen de } \\
\text { forma clara en la portada; todas las operaciones de } \\
\text { navegación se presentan de forma comprensible; hay } \\
\text { espacios en blanco alrededor de los textos. }\end{array}$ \\
\hline 8 & $\begin{array}{l}\text { Ayudar a los usuarios a } \\
\text { reconocer y diagnosticar y } \\
\text { recuperar errores }\end{array}$ & $\begin{array}{l}\text { Ayuda y documentación } \\
\text { colostructura del cibermedio es simple y clara; los } \\
\text { agradable; los menús son breves y se comunican; los } \\
\text { botones se destacan del fondo donde están inserta- } \\
\text { dos en esa plataforma. }\end{array}$ \\
\hline $\begin{array}{l}\text { Ayuda online; la función de ayuda está visible en ese } \\
\text { cibermedio. }\end{array}$ \\
\hline $\begin{array}{l}\text { Está disponible un mapa del sitio u otro recurso de } \\
\text { ayuda en la plataforma. }\end{array}$ \\
\hline 10
\end{tabular}

Al concluir este proceso, las notas de los expertos fueron recopiladas en una lista, organizando los problemas de acuerdo a los principios que fueron violados y también con cuál grado de severidad. Todos los problemas encontrados fueron clasificados por los expertos en una escala de severidad de 0 a 4 con los siguientes valores: 0 - Ningún problema de usabilidad; 1 - Problema Leve, corrección cuando tenga tiempo extra; 2 - Problema Medio, con poca prioridad para corrección; 3 - Problema Grave, con alta prioridad de corrección; 4 - Problema Muy Grave, con corrección obligatoria. Una vez que la evaluación fue completada, los diferentes resultados fueron comparados. Las fechas de los análisis correspondieran a los meses de junio y julio de 2018.

\section{Resultados y Discusión}

Los resultados posibilitaron detectar los problemas de usabilidad de la interface del portal MCTIC, analizada de acuerdo con los principios de usabilidad propuestos en la lista de heurísticas presentada en este estudio.

A partir de la evaluación de los expertos, fueron detectados siete problemas de usabilidad en la interface de esta plataforma. El mayor número de problemas correspondió a la heurística "visibilidad del status del sistema", que presentó tres problemas de usabilidad. Los expertos también encontraron problemas en las heurísticas "flexibilidad y eficiencia de uso", "consistencia y estándares", "reconocimiento en lugar de recuerdo" y "diseño minimalista y estético". En cada una de esas heurísticas, fue detectado un problema de usabilidad. A cada problema detectado, el experto 
atribuyó un grado de severidad. De forma que, los siete problemas de usabilidad encontrados fueron distribuidos en distintos niveles de gravedad. Los tres problemas de usabilidad detectados en la heurística "visibilidad del status del sistema" presentaron distintos niveles de gravedad. El primer problema fue clasificado en un nivel 3 (grave) y el según y el tercero en un nivel 2 (medio).

El primer problema de la "visibilidad del status del sistema" correspondió a la información presentada en la interface del cibermedio. Para los expertos 1 y 2 la información sobre la presencia de documentos de acciones del Ministerio en las áreas de ciencia y tecnología no es clara, no manteniendo al usuario informado sobre la localización de los contenidos y tampoco está claro lo que sucede en cada paso de la plataforma.

Dentro de la heurística de "visibilidad del status del sistema" encontramos que el segundo problema está relacionado con el feedback del portal, más concretamente, con la terminología usada en el Menú de la página principal. De acuerdo con el experto 2, las secciones del Menú para acceso a los videos y audios sobre las actividades del MCTIC, deberían presentar una subsección con un título más específico. De esa manera, el usuario podría acceder directamente a los videos y audios que corresponden a las acciones específicas del Ministerio, los cuales son difíciles de identificar, tal y como está actualmente presentados en el Menú, ya que se encuentran mezclados con otros tipos de videos de centros de ciencia, universidades y fundaciones públicas.

El usuario sólo tiene conocimiento que puede acceder a secciones específicas de cada institución, cuando accede a la página siguiente del portal. De esa manera el usuario es obligado a hacer un largo camino hasta completar esta tarea. Para los usuarios con poca experiencia con informática, puede no estar claro que esta sección no dispone de enlaces para contenidos de otras instituciones que no sean el Ministerio.

Similar a lo que paso en el Menú, en el Mapa Web, la sección para al acceso a las imágenes (fotos) también presentó problemas de usabilidad. Nuevamente, el experto 2 consideró que debería ser presentada una subsección con un título que sea más intuitivo, que permita al usuario acceder a las imágenes de las distintas instituciones más fácilmente. Para tener acceso a las imágenes de las acciones de las fundaciones públicas o centros de investigación, los usuarios son obligados a recorrer al buscador de la plataforma.

El ultimo problema dentro de la "visibilidad del status del sistema" también correspondió al feedback disponible. El experto 3 clasificó con un nivel 2 de gravedad (medio), problemas encontrados en tres secciones en el sitio web. En las secciones Ouvidoria (Oidoria), Acesso a Informação (Acceso a la Información) y Dados Abertos (Datos Abiertos) no está claro para los usuarios la posibilidad de hacer cambios para la obtención de más información. Los usuarios sólo tienen conocimiento que pueden hacer pequeños cambios para nuevas informaciones, cuando el cursor pasa por arriba de los contenidos.

En lo que respeta la heurística "flexibilidad y eficiencia del uso", los expertos 1, 2 y 3 encontraron un problema de usabilidad, con nivel 3 de gravedad (grave). En dos secciones del menú principal no fue posible la interacción con la aplicación, específicamente, en lo que respeta al comando Ctrl+, para aumento del tamaño de la fuente del texto. Este comando no tuvo efecto en las imágenes, apenas en las páginas donde están presentadas. Por el hecho de no posibilitar la interacción del usuario con la aplicación, a través del aumento del tamaño de la fuente del texto (Ctrl+), fue detectado un problema de usabilidad, correspondiente a la heurística "consistencia y estándares". De acuerdo con los expertos 1,2 y 3 , el sistema no sigue las convenciones y expectativas de los usuarios, también clasificando ese problema con nivel 3 de gravedad (grave).

En lo que respeta a la heurística "reconocimiento en lugar de recuerdo", el experto 3 detectó un 
problema con nivel 2 de gravedad (medio). El evaluador consideró que las opciones de navegación y los links para acceder los contenidos presentados en el sitio web, específicamente, la sección con el título Indicadores (Indicadores), no son presentados de forma clara y comprensible para el usuario.

El último problema de usabilidad encontrado en la plataforma analizada correspondió a la heurística "diseño minimalista y estético", también siendo clasificado con nivel 2 de gravedad. De acuerdo con el experto 2, algunos contenidos no se adaptan al tamaño del monitor, no permitiendo ver todo el contenido verticalmente.

\section{Conclusión}

Este estudio tuvo como objetivo el análisis de la situación de la divulgación de la ciencia en Brasil. El gobierno de Brasil realizó varios esfuerzos en este sentido, siendo el Ministerio de la Ciencia, Tecnología, Innovación y Comunicación, el MCTIC, la institución encargada de la popularización de la ciencia en el país. Una prueba de esos esfuerzos por parte de esta institución fue la creación de un completo portal web para popularizar la ciencia, disponibilizando gran cantidad y variedad de datos, estudios estadísticos, noticias, etc.

En este trabajo investigamos uno de los parámetros más importantes para la evaluación de la efectividad para divulgar y promover la información de esta página web: su usabilidad. Así, este artículo se centró en hacer una evaluación del acceso a los contenidos y servicios del portal MCTIC, centrándonos, en particular, en la interface de esa plataforma. La evaluación se basó en uno de los métodos más aceptados en el mundo académico: el Método de Evaluación Heurística propuesto por Nielsen (2016b).

El objetivo de este trabajo fue alcanzado y los resultados obtenidos de este análisis posibilitaron detectar algunos problemas de usabilidad de su interface en diferentes aspectos y con distintos niveles de gravedad. El mayor numero de problemas respeta a la "visibilidad del status del sistema", donde fueron encontrados problemas considerados graves en la disponibilidad de la información y feedback de esta plataforma. Además, las heurísticas "flexibilidad y eficiencia de uso", "consistencia y estándares", "reconocimiento en lugar de recuerdo" y "diseño minimalista y estético" también no estaban en acuerdo con los principios de usabilidad propuestos por Nielsen (2016b).

Así, a pesar de que este portal presenta contenidos y servicios de gran valor al ciudadano, el acceso a información científica a través de la interface de esta plataforma se mostró insatisfactorio en varios aspectos. La conceptualización de esta plataforma debe estar basada en sistemas que atiendan a diversos perfiles de usuarios, prestando especial atención al público no científico.

El portal de MCTIC debe proporcionar recursos que apoyen y conduzcan a los visitantes sin ningún o poco conocimiento de ciencia durante la toda navegación. Esto no siempre ocurre, tal como indicaron los resultados de este estudio. En concreto, se identificó un uso de terminología no adecuada para el público menos familiarizado con el portal y con la ciencia dentro de la heurística de "visibilidad del status del sistema". También el portal investigado sufre de uno de los errores más frecuentes a la hora de divulgar temas científicos: el de presentar informaciones de ciencia pero no presentar suficientes entornos para acceder a esa información.

Por lo tanto, una mejora en la usabilidad de la plataforma analizada facilitaría su uso y por consiguiente su efectividad a la hora de transmitir la información de ciencia en Brasil. Deberían ser priorizados mecanismos de navegación que atiendan a los diversos perfiles de usuarios de forma que puedan reconocer e identificar los excelentes recursos disponibilizados por el Ministerio a través de su página web. 
Nuestro estudio pone de manifiesto que una mayor flexibilidad y eficiencia en el Menú mejoraría significativamente la experiencia del usuario. Pensamos que para una mejor divulgación de la ciencia en Brasil, es importante no sólo que los contenidos científicos estén disponibles, sino que también sean de fácil acceso y se presenten de forma atractiva. En este sentido creemos que una mejora de la usabilidad de la plataforma del MCTIC estimularía al usuario científico y no científico en la adquisición de conocimientos sobre ciencia y por lo tanto ayudaría a la divulgación y popularización de la ciencia en Brasil.

Dado el carácter social del análisis desarrollado en este artículo, una extensión natural de este trabajo es el estudio de usabilidad considerando también a los ciudadanos que tienen alguna deficiencia, como la ceguera, sordera o problemas de psicomotricidad (Sánchez-Labella, Simelio y Moreno-Sardá, 2017). Este asunto requiere nuevos estudios y se deja como una interesante línea de investigación para un trabajo futuro.

\section{Bibliografía}

Almeida, C.; Amorim, L.; Brito, F. Ferreira, J.F. ; Massarani, L. (Orgs.). (2015). Centros e museus de ciência do Brasil. Rio de Janeiro : Associação Brasileira de Centros e Museus de Ciência : UFRJ. FCC. Casa da Ciência ; Fiocruz. Museu da Vida. Recuperado de http://www.casadaciencia.ufri.br/ Publicacoes/guia/Files/guiacentrosciencia2015.pdf

Bodmer, W. F. (1985). The Public Understanding of Science. London: The Royal Society.

Bohman, J. (2004). Expanding dialogue: The Internet, the public sphere and prospects for transnational democracy. The sociologial review. Oxford. v. 52, n. 1 p. 131-155. DOI: $10.1111 / 1467-$ 954X.2004.00477

Brasil. (2016). Estratégia Nacional de ciência, Tecnologia e Inovação 2016-2020. Brasília: Ministério da Ciência, Tecnologia, Inovação e Comunicação - MCTIC. Recuperado de http://www.finep. gov.br/images/a-finep/Politica/16 032018 Estrategia Nacional de Ciencia Tecnologia e Inovacao 2016 2022.pdf

Brasil. (2011) Estratégia Nacional de Ciência, Tecnologia e Inovação 2012 - 2015: Balanço das Atividades Estruturantes. Brasília: Ministério da Ciência e Tecnologia e Inovação. Recuperado de http://www.mct.gov.br/upd blob/0218/218981.pdf

Dahlberg, L. (2001). Democracy via Cyberspace. New Media \& Society, v.3, n. 2, p.157-177. DOI: $10.1177 / 14614440122226038$.

Dahlgren, P. (2005). The Internet, Public Spheres, and Political Communication: Dispersion and Deliberation, Political Communication, v. 22, n. 2, p. 147-162. DOI: $10.1080 / 10584600590933160$

Delice, E. K.; Gungor, Z. (2009).The usability analysis with heuristic evaluation and analytic hierarchy process. International journal of industrial ergonomics,39(6); $934 \mathrm{i} 939$.

Di Gennaro, C; Dutton, W. (2006). The Internet and the public: Online and offline political participation in the United Kingdom. Parliamentary Affairs, v. 2, n. 59, p. 299-313. Recuperado de https://academic.oup.com/pa/issue/59/2

Escobar, Herton. (2018). Divulgação científica: faça agora ou cale-se para sempre. In ComCiência e 
divulgação científica. Carlos Vogt, Marina Gomes, Ricardo Muniz (Organizadores). Campinas, SP: BCCL/ UNICAMP.

Ferrer F.; Santos, P. (Orgs.). (2004). E-government: governo eletrônico no Brasil. São Paulo: Saraiva.

Hassan, Y., Martín Fernández, F. J. \& Iazza, G. (2004). Diseño Web Centrado en el Usuario: Usabilidad y Arquitectura de la Información. Hipertext. net, 2. Recuperado de http://www. hipertext.net/web/pag206.htm

ISO/IEC. 2001. ISO/IEC 9126-1:2001. (2001). Systems and Software engineering - Systems and Software Quality Requirements (SQuaRE) - System and Software quality models, International Organization for Standardization. Recuperado de http://www.iso.org/iso/catalogue detail. htm? csnumber $=22749$

ISO/IEC. 2008. ISO/IEC 25012. (2008). Systems and software engineering -- Systems and software Quality Requirements and Evaluation (SQuaRE) -- System and software quality models. International Organization for Standardization. Recuperado de http://www.iso.org/iso/catalogue detail.htm?csnumber $=35736$

ISO/IEC. 2010. ISO/IEC 9241-210:2010.(2010). Systems and Software engineering - Systems and Software Quality Requirements (SQuaRE) - System and Software quality models, International Organization for Standardization. Recuperado de https://www.iso.org/standard/52075.html Access October 2017.

ISO/IEC. 2011. ISO/IEC 2501. (2011).Systems and software engineering -- Systems and software Quality Requirements and Evaluation (SQuaRE) -- System and software quality models. International Organization for Standardization. Recuperado de https://www.iso.org/obp/ ui/\#iso:std:iso-iec:25010:ed-1:v1:en

Gang, D. B.; Gant, J. P. (Org) (2002a). Enhancig e-service delivery. State web portal: delivering and financing e-service. Arlington: PWC p. 5-34.

Gant, J.P; Gant, D.B. (2002b). Web Portal Functionality and State Government E-Services. Systen Science, HICSS. Proceedings of the 35th Hawaii International Conference on Systems Sciences, p. 1627 1636.

Gomes, W. (2018). A Democracia no Mundo Digital. São Paulo: Sesc.

Gonçalves, T. (2018). La e-participación y la implicación del público en las acciones institucionales de ciencia en Brasil y Portugal, Sphera Publica, 1 (18), 148-165.

Lee Younghwa, Kozar Kenneth A. (2012). Understanding of website usability: Specifying and measuring constructs and their relationships. Decision Support Systems. 52(2):450-463. Recuperado de http://www.sciencedirect.com/science/article/pii/S0167923611001679

Massarani, L. (2002). Ciência e público: caminhos da divulgação científica no Brasil. Massarani, L.; Moreira, I. de C.; Brito , F (Org.). Forum de Ciência e Cultura. Série Terra Incógnita,1(7), 232-250. Rio de Janeiro: Casa da Ciência.

Moreira, I. de C. (2006). A inclusão social e a popularização da ciência e tecnologia no Brasil. 
Revista Inclusão Social - IBICT, 1 (2), 25-40. Recuperado de http://revista.ibict.br/inclusao/article/ view/1512/1707

Navas, A., Marandino, M. (2009). Dimensión política de la popularización de la ciencia y la tecnología en América Latina. El caso de Brasil. Ciencias unam, 9 (6),48-56.

Nielsen, J. (2016a). The Distribution of Users' Computer Skills: Worse Than You Think. Recuperado de: https://www.n ngroup.com/articles/computer-skill-levels/

Nielsen, Jakob. (2016b). Web UX 2016 vs 2004. Study of 12 years' evolution in web usability. UX Conference in London. Recuperado de https://www.nngroup.com/videos/web-ux/

Nielsen, Jakob. (2012). Usability 101: Introduction to Usability. Recuperado de https://www. nngroup.com/articles/usability-101-introduction-to-usability/

Nielsen, J. (2000). Usabilidad. Diseño de páginas Web. Prentice Hall.

Nielsen, J. (1994). Enhancing the explanatory power of usability heuristics. Proc. ACM CH'I94 Conf. Boston, April 24-28, 152-158.

Pearrow, M. (2000). Web Site Usability Handbook with Cdrom. Charles River Media, Inc.

Quivy, R. Van Campenhoudt, L. (2005). Manual de investigación en Ciencias Sociales. Mexico: Limusa.

Sánchez-Labella Martín, I., Simelio, N., \& Moreno-Sardá, A. (2017). El acceso web para personas con capacidades limitadas en los ayuntamientos españoles. Cuadernos.Info, (41), 155-173. DOI: https://doi.org/10.7764/cdi.41.1061

Krug, S. (2013). Don't Make Me Think, Revisited: A Common Sense Approach to Web Usability (3ed) Berkeley: New Riders Publishing.

Vogt, C.; Gomes, M.; Muniz, R (Org). (2018). ComCiência e divulgação científica. Campinas, SP: BCCL/ UNICAMP.

Winter E. (2004). Public communication of science and technology: German and european perspectives. Science Communication, v.25, n.3, p. 288-293. DOI: $10.1177 / 1075547003262665$. 
MHCJ nº 10 (1) | Año 2019 - Artículo nº 11 (146) - Páginas 223 a 237 - mhjournal.org

\section{(c) (i) (2)}

Licencia Creative Commons

Miguel Hernández Communication Journal

mhjournal.org

\section{Cómo citar este texto:}

Tatiana Santos Gonçalves (2019): Comunicación y divulgación de ciencia en Brasil: un estudio de la usabilidad del portal MCTIC, en Miguel Hernández Communication Journal, n¹0 (1), pp. 223 a 237. Universidad Miguel Hernández, UMH (Elche-Alicante). DOI: http://dx.doi.org/10.21134/mhcj.v10i0.293 